\title{
SUCCESSFUL MANAGEMENT OF SACRAL POSTHERPETIC NEURALGIA WITH GANGLION IMPAR BLOCK - A CASE REPORT
}

\author{
PARTHASARATHY S, ANWAR BATCHA S
}

Department of Anesthesiology, Mahatma Gandhi Medical College and Research Institute, Sri Balaji Vidyapeeth University, Puducherry, India. Email: painfreepartha@gmail.com

Received: 06 October 2018, Revised and Accepted: 10 October 2018

\section{ABSTRACT}

Postherpetic neuralgia (PHN) is one of the most common and important complications of severe varicella-zoster infection, especially occurring in $20 \%$ of the elderly patients. A 59-year-old female presented with pain in the right sacral region for the past 3 months. The pain was preceded by herpes zoster. She was diagnosed as a case of Sacral PHN. We administered paramedian approach of ganglion impar block with $10 \mathrm{ml}$ of $0.25 \%$ bupivacaine with $8 \mathrm{mg}$ dexamethasone which resulted in immediate adequate pain relief. On follow-up after 8 weeks, the pain score continued to be less with 3/10. No complications were encountered. There was no sensory loss. We conclude that ganglion impar block with a combination of local anesthetic and steroid is a viable treatment option for sacral PHN.

Keywords: Chronic pain, Sacral, Postherpetic neuralgia, Ganglion impar block

(C) 2019 The Authors. Published by Innovare Academic Sciences Pvt Ltd. This is an open access article under the CC BY license (http://creativecommons. org/licenses/by/4. 0/) DOI: http://dx.doi.org/10.22159/ajpcr.2019.v12i2.29423

\section{INTRODUCTION}

Postherpetic neuralgia (PHN) is one of the most resistant chronic pain problems, usually affecting elderly patients. The pain of PHN usually follows the typical dermatomal distribution of the erythematous rash earlier caused by herpes zoster. Commonly unilateral thoracic dermatomes and the trigeminal nerve, especially the ophthalmic branch, are frequently affected [1], but sacral dermatome is not frequent. Despite adequate treatment, $20 \%$ suffer from the pain of the disease. Anticonvulsants, sympathetic, epidural blocks, and blocks of ganglion impar have been used with variable success [2].

Tricyclic antidepressants provide the best chance of relief with the few side effects. This is given along with anticonvulsant (carbamazepine, valproic acid, or phenytoin), but the possibility of liver dysfunction or bone marrow depression should be kept in mind. Gabapentin is effective for the management of PHN. It does not carry the risk of organ dysfunction which is seen with older anticonvulsants. Opioids may cause constipation in older patients with few benefits. Most studies fail to show much benefit from spinal cord stimulation. Transcutaneous electrical nerve stimulation (TENS) may help in occasional patients, but cause aggravation of pain in patients with severe allodynia, and is not beneficial if there is considerable cutaneous sensory loss. Topical capsaicin is occasionally helpful but can aggravate the pain in some individuals $[3,4]$. Transdermal lidocaine patches can be helpful in some patients. The ganglion impar [5] (ganglion of Walther) is the most caudal part of the sympathetic trunks. The lowest two sympathetic ganglia of the pelvis often fuse forming one ganglion in the midline anterior to the coccyx. We wished to block the sympathetic ganglion to get the desired pain relief.

\section{CASE REPORT}

A 59-year-old female came to our hospital with the complaints of pain in the right sacral region, which was constant, neuropathic in nature, gradually increased in intensity for the past 3 months. Earlier, she had clinical features of herpes zoster. She was treated with carbamazepine, topical acyclovir patches, and neurovitamins, after 3 weeks. A combination therapy with gabapentin and amitriptyline was changed for 3 weeks. Oral tramadol was added after 20 days. The combination of all these drugs produced less relief. The daytime sedation was too high to continue such combination. There was no improvement in pain. There was a mild hyperalgesia on touching the lesions (Fig. 1).

The visual analog score (VAS) remained at $7 / 10$. She did not have any other lesions over genitals, skin, and mouth. We administered ganglion impar block with $10 \mathrm{ml}$ of $0.25 \%$ bupivacaine with $8 \mathrm{mg}$ dexamethasone by Raj's paramedian approach. After $20 \mathrm{~min}$, the patient was pain free (VAS - 1-2/10). There was no sensory loss. After 4 weeks, the patient came for follow-up and the pain score continued to be less with a VAS score of $3 / 10$. No complications were noted. The only carbamazepine regimen of $200 \mathrm{mg}$ in the night was continued.

\section{DISCUSSION}

Varicella-zoster virus, a member of the herpes virus family, is a significant cause of morbidity despite immunization. The incidence of varicella (chickenpox) is over 4 million cases per year, whereas the incidence of herpes zoster is 300,000 cases per year. Primary infection with varicellazoster virus results in varicella (i.e., chickenpox) which is followed by a latent phase where the virus remains sequestered in dorsal root ganglia of the trigeminal ganglion remaining dormant; however, it may be reactivated anytime, leading to herpes zoster (shingles). Reactivation may be triggered by infection, immunosuppression, trauma, irradiation, malignancy, and advancing age. Herpes zoster is characterized as severe pain in one or more dermatomes usually followed by a vesicular skin eruption 7-10 days later. Persistence of painful symptoms for 4-6 weeks after the termination of the acute phase of infection is referred to as PHN. The prevalence of PHN is around $9-45 \%$ of all cases of herpes zoster [6]. Early institution of sympathetic blockade has been advocated to decrease the incidence and severity of PHN [7]. This therapy remains controversial because a direct correlation between sympathetic blockade and prevention of PHN has not been proven by randomized prospective trials. The ganglion impar is the fused terminal end of the paravertebral sympathetic chain and is found at the level of the sacrococcygeal junction. Neural blockade of the ganglion impar has been advocated as a means of managing intractable pelvic and perineal pain in cancer patients [8]. A review of the literature reveals very limited information on the usefulness of blockade of the ganglion impar for pain other than that of neoplastic origin. The original technique for blockade of the ganglion impar described by Plancarte et al. [9] involves a midline 


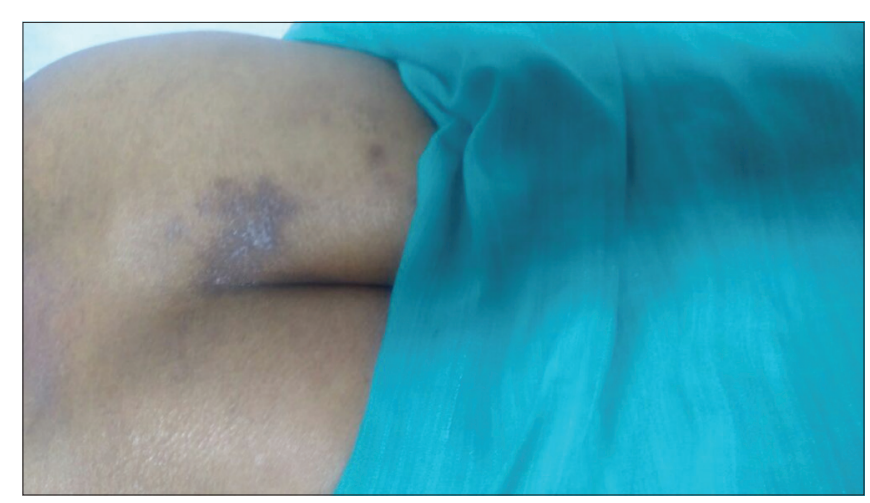

Fig. 1: Sacral postherpetic lesions

approach through the anococcygeal ligament with advancement of the needle tip cephalad to the anterior surface of the sacrococcygeal ligament. This technique involves placing one or two bends near the tip of the needle to more easily approximate the location of the ganglion. However, a gently curved needle has also been advocated to negotiate around the natural curve of the coccyx. Insertion of a finger in the rectum has been advocated as a safeguard against perforation of the rectum because it lies in close proximity to the solitary ganglion. This technique can be quite uncomfortable in a patient with rectal pathology and also makes it difficult to maintain sterility during the procedure. Wemm Jr. and Saberski [10] advocate passing directly through the sacrococcygeal ligament to reach the ganglion impar. This approach can be useful in patients with normal anatomy but may prove challenging in patients with arthritic changes in the bones and calcification of the ligaments of the sacrum and coccyx. Raj et al. [8] briefly described a paramedian approach to the ganglion which was administered by us. Huang [11] recently described a lateral approach to the ganglion approaching from below the transverse process of the coccyx inferior to the level of the sacrococcygeal junction. The pain caused by PHN may involve both peripheral and central mechanisms. This understanding likely explains why PHN is so difficult to treat when the lesion is not confined to an accessible dermatome like intercostal nerve where we can administer the selective intercostal nerve block with ease. However, when the lesion is in remote area-like sacral region where targeting a single nerve is not possible, we planned to target the sympathetic component as was done in our case. It also stresses the importance of multiple modes of therapy. The role of corticosteroids [12] in pain relief for this patient is uncertain. Corticosteroid injections with nerve blocks have had unpredictable and limited success in the treatment of PHN. Sacral dermatomal distribution of zoster and PHN is unusual but can lead to severe and disabling pain. Blockade of the ganglion impar may play a significant role in the treatment of this disabling pain. Further, investigation is needed to determine efficacy of this sympathetic block in the treatment of the pain of zoster and PHN in the sacral dermatomes. Due to the low incidence of complications with blockade of the ganglion impar, it may have a role in early aggressive therapy of zoster in the sacral dermatomes. As such, there are only few case reports [13] in literature in the management of sacral PHN by ganglion impar block. Hence, this case is reported for its rarity. The commonly used outpatient opioid is tramadol, and the use of the same drug to counter pain in PHN may not be predictable in all cases due to genetic reasons $[14,15]$. By blocking the sympathetics, we can overcome the problem mentioned above. Even though ultrasound [16] can help in administration of the block, we gave it by the classical approach only.

\section{CONCLUSION}

We conclude that ganglion impar blockade is a viable treatment option for sacral PHN. As the involved sacral region is not amenable to be easily targeted like intercostal area, the blockade of ganglion impar is a definite therapeutic modality.

\section{CONFLICTS OF INTEREST}

The authors declare that they have no conflicts of interest.

\section{REFERENCES}

1. Gauthier A, Breuer J, Carrington D, Martin M, Rémy V. Epidemiology and cost of herpes zoster and post-herpetic neuralgia in the United Kingdom. Epidemiol Infect 2009;137:38-47.

2. Dworkin RH, Schmader KE. Treatment and prevention of postherpetic neuralgia. Clin Infect Dis 2003;36:877-82.

3. Weller TH. Varicella and herpes zoster: A perspective and overview. J Infect Dis 1992;166 Suppl 1:S1-6.

4. Cunningham AL, Dworkin RH. The management of post-herpetic neuralgia. BMJ 2000;321:778-9.

5. Waldman $\mathrm{SD}$, editor. Hypogastric plexus block and impar ganglion block. In: Pain Management. Philadelphia, PA: Saunders/Elsevier; 2007. p. 1354-7.

6. Bowsher D. Post-herpetic neuralgia in older patients. Incidence and optimal treatment. Drugs Aging 1994;5:411-8.

7. Winnie AP, Hartwell PW. Relationship between time of treatment of acute herpes zoster with sympathetic blockade and prevention of postherpetic neuralgia: Clinical support for a new theory of the mechanism by which sympathetic blockade provides therapeutic benefit. Reg Anesth 1993;18:277-82.

8. Raj PP, Lou L, Erdine S, Staats PS. Ganglion of impar block. In: Raj PP, editor. Radiographic Imaging for Regional Anesthesia and Pain Management. New York: Churchill Livingstone; 2003. p. 238-41.

9. Plancarte R, Amescua C, Patt RB, Allende S. Presacral blockade of the ganglion of Walter (ganglion impar). Anesthesiology 1990;73:A751.

10. Wemm K Jr., Saberski L. Modified approach to block the ganglion impar (ganglion of walther). Reg Anesth 1995;20:544-5.

11. Huang JJ. Another modified approach to the ganglion of walther block (ganglion of impar). J Clin Anesth 2003;15:282-3.

12. Pasqualucci A, Pasqualucci V, Galla F, De Angelis V, Marzocchi V, Colussi R, et al. Prevention of post-herpetic neuralgia: Acyclovir and prednisolone versus epidural local anesthetic and methylprednisolone. Acta Anaesthesiol Scand 2000;44:910-8.

13. McAllister RK, Carpentier BW, Malkuch G. Sacral postherpetic neuralgia and successful treatment using a paramedial approach to the ganglion impar. Anesthesiology 2004;101:1472-4

14. Nasare N, Banerjee B, Deshmukh P, Mediratta P, Ahmed R, Saxena A, et al. The impact of pharmacogenetics on adverse drug reactions to predict the efficacy of tramadol monotherapy for the treatment of post herpetic neuralgia patients. Int J Pharm Pharm Sci 2014;6:89-96.

15. Nasare N, Mediratta P, Banerjee B, Deshmukh P, Saxena A, Bhattacharya $\mathrm{S}$, et al. Assessment of cyp $2 \mathrm{~d} 6 * 10$ polymorphism with post herpetic neuralgia patients undergoing tramadol treatment. Int $\mathrm{J}$ Pharm Pharm Sci 2014;6:97-102.

16. Lin CS, Cheng JK, Hsu YW, Chen CC, Lao HC, Huang CJ, et al. Ultrasound-guided ganglion impar block: A technical report. Pain Med 2010;11:390-4. 